
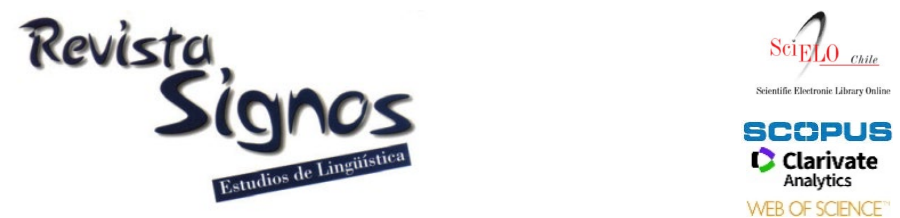

\title{
Introducción a la sección monográfica: Estudios de Lingüística Sistémico-Funcional en/del español
}

\section{In Memoriam}

Dedicamos este volumen monográfico a la memoria de nuestros queridos colegas, Elsa Ghio y Giovanni Parodi, insignes lingüistas latinoamericanos, prematuramente fallecidos en 2020. La fecunda obra de Elsa Ghio constituye de alguna manera, el germen de las contribuciones recogidas en este volumen, el cual no habría visto la luz sin el entusiasta y permanente apoyo de Giovanni Parodi.

\section{INTRODUCCIÓN}

La Lingüística Sistémico Funcional (LSF), heredera entre otras fuentes de la Escuela de Praga, lleva siendo una valiosa herramienta para la comprensión y el estudio del lenguaje desde los años 60 del pasado siglo. Inspirado, además de por la citada Escuela de Praga, por figuras como Malinovski, Bernstein y, más directamente, su maestro Firth, Michael Halliday fue el fundador y gran impulsor de esta interpretación del sistema semiótico humano como medio para construir la experiencia de nuestro mundo interior y exterior, siempre como reflejo del contexto social en el que se desarrolla. Aunque una gran parte de los estudios que han utilizado la LSF se han centrado en el inglés, lo que le ha costado la inmerecida crítica de ser una teoría anglo-céntrica, ${ }^{1}$ existe también una rica tradición de aplicación de la teoría al estudio de otras lenguas, entre ellas el español.

Al igual que la Introduction to Functional Grammar de Halliday (1985 en su primera edición) se considera la primera gran obra de referencia de la LSF en general, y para el inglés en particular, el Manual de Lingüistica Sistémico Funcional de Elsa Ghio y María Delia Fernández (2005) puede ser tomado como la primera gran obra de referencia de la LSF por lo que respecta al español y ciertamente sirve como introducción a la teoría para los hispano-parlantes, al estar además escrito en español. Aquellos que no estén 
familiarizados con la LSF tienen, pues, en ese manual el punto de partida idóneo para adentrarse en el mundo de la sistémica.

Otra obra de referencia sobre la LSF en español es la gramática contrastiva español/inglés de Lavid, Arús y Zamorano (2010), donde se describen los recursos léxico-gramaticales del español y se especifican aquellas áreas de la léxico-gramática en las que el español y el inglés contrastan de manera más clara. A diferencia del Manual de Ghio y Fernández (2005), esta gramática está escrita en inglés, por lo que resulta menos accesible para aquellos sin destrezas de lectura en esa lengua.

Precisamente para comenzar a paliar los problemas de acceso de los hispanoparlantes a las fuentes sistémicas esenciales, salió a la luz en 2017 el volumen Obras esenciales de M.A.K. Halliday, compilado por Elsa Ghio, Federico Navarro y Annabelle Lukin. Aunque esta colección de traducciones de artículos del fundador de la LSF no contribuye directamente a la aplicación de la teoría al español, sí que supone una valiosa fuente teórica para la LSF en español, como lo es también el libro Leer para Aprender, traducción a cargo de Ana Bustelo (2018) de la obra de Rose y Martin (2012), Learning to Write, Reading to Learn.

Aparte de las obras de referencia recién mencionadas, existe un amplio elenco de publicaciones sobre distintos aspectos del español, tanto de forma aislada como en comparación con otras lenguas, provenientes de investigadores de nuestra lengua en diversas partes del mundo, sobre todo, como es lógico, países de habla hispana. Aun a riesgo de ser demasiado generalistas e ignorar de forma injusta algunas importantes contribuciones a la LSF en y del español, se pueden distinguir seis focos geográficos principales, cada uno con sus características específicas; a saber, y por orden alfabético, Argentina, Chile, Colombia, España, México y Venezuela.

Argentina y España fueron de entre las primeras en incorporar la LSF a la rica tradición de estudios lingüísticos del español. La ya mencionada Elsa Ghio fue una de las responsables del despegue de la LSF en Argentina, trabajando desde la Universidad del Litoral en aplicaciones de la teoría sistémica a la enseñanza y a diversas cuestiones discursivas. Como consecuencia, la aplicación de la LSF a estos dos campos ha sido muy fructífera en Argentina en los últimos años, lo cual se ve reflejado en este volumen, en el que varios autores argentinos tratan de cuestiones discursivas y de enseñanza.

En España la tradición sistémica comenzó fuera del área del español, gracias a los trabajos sobre el inglés de Angela Downing en la Universidad Complutense de Madrid desde los años 90 del pasado siglo. A partir de ahí surgieron varias iniciativas de investigación dentro del marco de la LSF con un marcado cariz contrastivo entre el inglés y el español, sobre todo a nivel de la léxico-gramática, ejemplo de lo cual es la antes mencionada gramática contrastiva español/inglés de Lavid et al. (2010). 
La LSF tiene también una larga y fértil tradición en México, donde, al igual que en Argentina, las aplicaciones pedagógicas y discursivas han sido preponderantes, con una más reciente inclinación hacia los estudios de valoración. El núcleo desde el que se ha extendido la LSF al resto del país es la Universidad Nacional Autónoma de México, donde en 2016 se publicó el libro, coordinado por Natalia Ignatieva y Daniel Rodríguez Vergara, Lingüística Sistémico Funcional en México: aplicaciones e implicaciones.

En Chile, diversos investigadores de la Pontificia Universidad Católica de Valparaíso, liderados por Giovanni Parodi, llevan varios años utilizando la LSF en estudios sobre los géneros discursivos. Igualmente, de carácter discursivo es la investigación que ha venido llevando a cabo el grupo de Teresa Oteíza en la Pontificia Universidad Católica de Chile, donde también son de gran relevancia los trabajos sobre la léxico-gramática del español, sobre todo a nivel interpersonal, llevados a cabo por Beatriz Quiroz.

La LSF en Colombia tiene su máximo exponente en la Universidad del Norte, Barranquilla, donde Gillian Moss y sus colaboradores han realizado importantes contribuciones en el área de la educación.

La LSF hizo su entrada en Venezuela en los años 80 a través de la Escuela de Idiomas Modernos de la Universidad Central de Venezuela, bajo la responsabilidad de Adriana Bolívar. Posteriormente, y con la colaboración de Martha Shiro, la LSF se introdujo en el nivel de postgrado, donde se desarrolló la investigación y se formaron nuevos profesores en la LSF, como Rebecca Beke y Nora Kaplan, quienes siguieron fortaleciendo esta perspectiva. La investigación en idioma español resultó en la elaboración de diversos corpus de temática variada.

No quiere decir este somero repaso de los principales focos de producción de LSF en y del español que no se encuentren contribuciones destacables en otras partes del mundo hispanoparlante. La LSF se aplica al estudio de nuestra lengua no sólo a lo largo y ancho de España y América Latina sino también en países en los que el español no es la primera lengua, como es el caso de los EE.UU de América, donde la Universidad de California en Davis es un referente en la aplicación de la LSF al español como lengua heredada, y de Canadá, desde donde Maite Taboada, de la Simon Frazer University, lleva años aportando estudios discursivos del español en contraste con el inglés.

Como consecuencia de la creciente importancia de los estudios sobre la lengua española y diversos aspectos socio-culturales relacionados con los hispanoparlantes desde el enfoque de la LSF, se constituyó en el año 2004 la asociación de Lingǘstica Sistémico Funcional de América Latina (ALSFAL), con la intención de que "nucleara a los lingüistas que trabajan bajo los aportes teóricos de la Lingüística SistémicoFuncional en los países de esa región" (https://alsfales.wordpress.com/sobre- 
nosotros/historia/). ALSFAL engloba investigadores no sólo del español sino también del portugués, al ser Brasil uno de los bastiones de la LSF en América Latina.

Tal vez por la distancia geográfica, la colaboración, e incluso la información, entre los lingüistas sistémicos de diversos puntos del mundo hispanoparlante no ha sido la deseable. Ese es probablemente uno de los retos principales de la LSF del y en español de cara al futuro y de ahí surgió la idea de organizar el primer coloquio de/en del español en la Universidad de Salamanca en el año 2017, con participantes tanto de España como de América Latina. Con ese mismo espíritu sale a la luz este volumen resultante en su mayoría de las contribuciones al congreso salmantino, con la incorporación de algunos colegas que no pudieron acompañarnos en aquel momento pero que no han querido perder la ocasión de sumarse a este proyecto de colaboración. El resultado son 8 artículos de enfoque marcadamente discursivo, con contribuciones de lingüistas afincados en Argentina, México, EE.UU. y Canadá.

El volumen se inicia con el artículo de María Belén Grisolía, en el que esta autora estudia interacciones socialmente polémicas, como son los mensajes automáticos (vía correos electrónicos, mensajes de texto y contestadores). Dichos mensajes suscitan en los usuarios la sensación de estar participando de 'inter'acciones en las que finalmente quedan hablando solos. Para ello, indaga sobre la distribución de la responsabilidad en el discurso, definida en términos de agentividad y modo. A partir del análisis de la combinación de recursos vinculados con la realización del principio de gradualidad en el diálogo con las realizaciones de los sistemas de modo y modalidad, Grisolía concluye que el elemento que mueve estratégicamente la aguja de la personalización a la impersonalización, y viceversa, en la organización discursiva, es el cambio de participante de la interacción.

Natalia Ignatieva explora la relación entre los significados ideacionales (también llamados 'ideativos', véase el artículo de Salvio Martín Menéndez) e interpersonales en los escritos de estudiantes universitarios de historia, con el objetivo específico de analizar la transitividad verbal y los aspectos valorativos en tres tipos de procesos (y sus respectivas cláusulas): verbales, mentales y relacionales. En su investigación, que combina tanto el análisis cuantitativo como el cualitativo y que forma parte de un proyecto más amplio sobre tipología verbal y evaluación en la escritura académica de las humanidades, Ignatieva contrasta dos géneros muy utilizados en la disciplina de historia: el ensayo y la pregunta-respuesta. Su análisis le permite concluir que la pertenencia de escritos a una misma disciplina parece tener un papel más decisivo en la selección de recursos lingüísticos a la hora de redactar que su pertenencia a un género discursivo determinado.

Dalia Magaña y Teenie Matlock analizan cómo los pacientes de cáncer, hombres y mujeres de habla hispana en Estados Unidos, utilizan la metáfora y la modalidad al compartir en un sitio en línea sus experiencias personales con la enfermedad. El 
estudio amplía un trabajo anterior centrado solo en las mujeres, al considerar las autoras que las diferencias de género son un factor relevante en el discurso sobre el cáncer. En este artículo se muestra cómo las mujeres usan metáforas para hablar sobre el cáncer con mayor frecuencia que los hombres. Por otra parte, tanto la frecuencia como los tipos de verbos modales que usan las mujeres y los hombres son distintos. Las mujeres tienden a establecer conexiones personales y mitigar las señales que podrían considerarse autoritarias. Los hombres, por su parte, se inclinan por recurrir más a la modulación, dando consejos y órdenes directas. Estos hallazgos, en línea con los de otros estudios realizados con angloparlantes, ayudarían a encontrar formas más efectivas de comunicarse y pensar sobre el cáncer.

Salvio Martín Menéndez, en un artículo de índole más teórica donde se replantea la categoría de agentividad, se propone demostrar que la organización gramatical de los rasgos que permiten caracterizar los procesos (realizados por las bases lexicales de los verbos) depende, en última instancia, de la organización estratégica de los discursos. Esto permitiría explicar el funcionamiento efectivo de los recursos gramaticales en relación con las estrategias de los que son parte constitutiva. Utilizando un ejemplo tomado de una serie discursiva de titulares de noticias de varios periódicos argentinos sobre las opiniones que el papa Francisco dio sobre los índices de desocupación en ese país, el autor analiza los procesos para determinar el alcance y necesaria complementación entre la agentividad gramatical (textual) y la discursiva. Concluye el autor planteando que la proyección discursiva de los procesos se debe llevar a cabo a partir de una caracterización de una gradualidad semántica que permite establecer rasgos que se combinan con grados de concreción, agentividad textual y discursiva.

A través del análisis de un amplio corpus de comentarios de profesores a los textos producidos por estudiantes que cursan asignaturas avanzadas de distintas carreras de grado, Lucía Natale examina cómo se realiza lingüísticamente la negociación sobre las epistemologías y las ideologías aceptadas en una cultura disciplinar. Los resultados muestran que el posicionamiento epistemológico e ideológico de los docentes así como la búsqueda de alineación de los estudiantes se lleva a cabo mediante varias estrategias discursivas diferenciadas, de las cuales este artículo analiza tres en particular: Rechazar, Ajustar y Problematizar. Desde la perspectiva de la Valoración, estas estrategias se inscriben en el sistema de Compromiso. Con la primera ED, se produce una contracción dialógica con la que los docentes/expertos se construyen como guardianes de la cultura interdisciplinar, impidiendo el paso de los novatos. En la segunda, los docentes matizan sus evaluaciones negativas con recursos de Gradación y dejan entrever al estudiante que su producción escrita académica puede ser mejorada a partir de su interacción con el docente/experto. Finalmente con la tercera ED, los docentes no clausuran totalmente la aserción del estudiante, sino que seleccionan recursos heteroglósicos mitigadores, ofreciéndole alternativas que le servirán para repensar sus interpretaciones. 
En el siguiente artículo, Mariana Pascual aborda un hecho social traumático, como fue la dictadura militar en Argentina (1974-1983) y la reacción social a la misma durante las dos décadas siguientes. Su propuesta, que deriva del análisis del discurso de medios argentinos en el período 1984-2004 tiene un doble propósito: por un lado, proponer una tipología de recursos léxico-gramaticales que construyen significados evaluativos en español para la construcción discursiva de los pasados traumáticos y, por el otro, establecer una correlación entre esa reacción social y un desarrollo sistemático gradual de los significados valorativos, lo que la autora denomina su Dinámica Evaluativa (DE). Los resultados reflejan una mutación en la construcción de los Juicios ya que a medida que se aleja en el tiempo el hecho social traumático de la dictadura se generan combinaciones de significados que incorporan elementos de Afecto, los que se fusionan para re-presentar cómo la sociedad enfrentó su pasado reciente y desarrolló su memoria colectiva.

Carolina Vieira y Maite Taboada indagan sobre las opciones teórico-metodológicas más adecuadas para analizar textos académicos orales de conferencias académicas (Cas) en español, dentro del marco de la LSF y, en particular, de la Teoría de Género y Registro. Las autoras analizan un corpus de ponencias en paneles de conferencias realizadas por académicos que trabajan en programas de español en los Estados Unidos. Su trabajo destaca la importancia de los elementos del lenguaje que manifiestan el propósito social de la vinculación con la audiencia y el posicionamiento del ponente ante el conocimiento colectivo de su comunidad académica. Los resultados muestran cómo la LSF puede usarse para entender este tipo de textos teniendo en cuenta la variación y su carácter interpersonal. Respecto a la determinación de su estructura genérica, las autoras señalan que entender las CAs como un macro-género es vital para un estudio cabal de las mismas. Por ello, la gran variabilidad de los textos del corpus puede resolverse al considerar las CAs como un macro-género de estructura orbital. Este estudio aporta al conocimiento sobre las CAs al discutir aspectos teóricos y metodológicos pertinentes desde la perspectiva de la LSF.

En el artículo final, Victoria Zamudio-Jasso se interesa por la manera en que el lenguaje evaluativo es utilizado en el discurso académico en español dentro del área de las humanidades, en particular, en el campo de los estudios literarios. Utilizando como marco teórico-analítico el Modelo de la Valoración y, en particular, los Sistemas de actitud y gradación, la autora compara la manera en que estudiantes universitarios de licenciatura, por un lado, y académicos por otro, expresan opiniones y actitudes en sus textos. Los resultados de su estudio muestran tanto similitudes como diferencias. Las similitudes se observan en aspectos más generales, como el uso preponderante de los recursos de gradación para aumentar la intensidad de las valoraciones inscritas, mientras que las diferencias tienen que ver especialmente con los recursos de extensión y el tipo de recursos léxicos y gramaticales que se prefieren. Este estudio 
comparativo tiene implicaciones pedagógicas, al posibilitar el guiar el desarrollo del estudiante como escritor de textos académicos hacia las prácticas más comunes y aceptadas en su disciplina.

Jorge Arús Hita (Universidad Complutense de Madrid)

Nora Kaplan (Universidad de Salamanca)

Editores invitados

\section{REFERENCIAS BIBLIOGRÁFICAS}

Bustelo, A. (2018). Leer para aprender (Traducción de Learning to Write: Reading to Learn [Jim Martin \& David Rose, 2012)]. Madrid: Pirámide.

Ghio, E., Navarro, F. \& Lukin, A. (2017). Obras esenciales de M.A.K. Halliday. Santa Fe: EdicionesUNL. $\quad$ https://drive.google.com/file/d/1zHpV LH7QCfE3cVhMHR7YNipl0_zANs/view

Ghio, E. \& Fernández, M.D. (2005). Manual de Lingüistica Sistémico Funcional: El enfoque de M. A. K. Halliday y Ruqaiya Hasan: Aplicaciones a la lengua española. Santa Fe, Argentina: Universidad Nacional del Litoral.

Halliday, M. A. K. (1985). An introduction to Functional Grammar. Londres: Edward Arnold.

Ignatieva, N. \& Rodríguez Vergara, D. (2016). Lingüistica Sistémico Funcional en México: Aplicaciones e implicaciones. Ciudad de México: Universidad Nacional Autónoma de México.

Lavid, J., Arús, J. \& Zamorano, J. R. (2010). Systemic Functional Description of Spanish. A Contrastive Study with English. Londres: Continuum.

Rose, D. \& Martin, J. (2012). Learning to write, Reading to learn: Genre, knowledge and Pedagogy in the Sydney School. Sheffield: Equinox Publishing.

\section{NOTA}

${ }^{1}$ Halliday, sinólogo, se inspiró en el chino a la hora de desarrollar algunas de las ideas claves de la Lingüística Sistémico Funcional. 